\title{
Motor skills interventions in children with developmental coordination disorder: A review study
}

\author{
Daehyoung Lee ${ }^{1}$, Rudolf Psotta ${ }^{2}$, and Milena Vagaja ${ }^{2}$ \\ ${ }^{I}$ Indiana University, Bloomington, IN, United States of America; and ${ }^{2}$ Faculty of Physical Culture, Palacký Univer- \\ sity Olomouc, Czech Republic
}

Children with developmental coordination disorder (DCD) demonstrate significant motor deficits that place them at risk for other health problems such as low physical activity (PA) and overweight/obesity. There is a need to update the effect of diverse movement-based interventions that use gross motor skills to improve psychomotor development of this population. Thus, the purpose of this review study was to investigate the characteristics of effective motor skills interventions for children with DCD, analyzing types of motor tasks, instructional strategies, and learning environment. Studies published between January 1994 and December 2015 were extracted based on inclusion criteria: (1) clear identification of DCD, (2) experimental study design with pre- and post-test, (3) age range of 5 to 12 years, (4) motor/movement-based intervention, and (5) publication in peer-reviewed journal. Of the 317 studies identified, only seven met the review criteria. Most studies showed significant improvements in various motor skills of participants with DCD such as manual dexterity, ball skills, and balance. Various types of movement-based interventions can improve motor skills in children with DCD even though the level of improvement differs from study to study. However, more rigorous intervention studies are needed to: (1) identify motor intervention best practices, (2) examine the sustainability of changes, and (3) examine the impact of the intervention on other physical, health, social, and emotional outcomes.

Keywords: developmental coordination disorder, motor skills, motor learning, intervention for children with DCD

\section{Introduction}

\section{Cause and diagnosis of the developmental coordi- nation disorder (DCD)}

Atypical brain development that affects learning new motor skills is generally proposed as a cause of DCD (Gilger \& Kaplan, 2001). The sensorimotor dysfunction produced in the central nervous system has also been believed as the main cause of general symptoms of DCD (Deng et al., 2013). Nevertheless, the cause of DCD is still unclear (Lichtenstein, Carlström, Råstam, Gillberg, \& Anckarsäter, 2010).

According to the American Psychiatric Association (APA) (2013), DCD occurs when "the level of motor coordination and learning is substantially below that expected given the individual's chronological age, intelligence, and opportunity for performing motor skills". The Diagnostic and Statistical Manual of Mental Disorders (APA, 2013) is a global standard for the diagnosis of DCD and includes four criterion relating to the motor performance, disturbance in daily living, onset of symptoms, and the relation to other medical conditions.
Significantly, the estimated prevalence of DCD is approximately 5-6\% in school-aged children (Blank, Smits-Engelsman, Polatajko, \& Wilson, 2012) and it can persist into adolescence and adulthood (Rasmussen \& Gillberg, 2000). As a result, DCD has been described as one of the six most commonly diagnosed developmental disorders (Kwan, Cairney, Hay, \& Faught, 2013).

\section{Manifestations of DCD}

Children with DCD typically show the delayed reaction time to sensory stimuli with decreased adaptability and flexibility in motor learning/motor behavior. In addition, they tend to rely heavily on visual feedback (Barnhart, Davenport, Epps, \& Nordquist, 2003) and demonstrate poorer visual spatial processing compared to typically developing (TD) children (Wilson \& McKenzie, 1998).

More importantly, movement and coordination problems are the main manifestations of DCD. Children with DCD often experience difficulties in playground activities such as hopping, jumping, running, and catching or kicking a ball. Poor coordination skills 
in academic activities including writing, drawing, and using scissors often result in scribbled and more childish academic performance (Dewey, Kaplan, Crawford, \& Wilson, 2002).

The representative outcome of DCD is a low level of PA. Children with DCD have a lower task and barrier efficacy toward PA than their peers and they spend significantly less time in moderate-to-vigorous PA than their peers (Batey et al., 2014; Cairney, Hay, Faught, Wade, et al., 2005). Also, children with DCD, in general, are less physically fit (Faught, Hay, Cairney, $\&$ Flouris, 2005) and remarkably exposed to overweight and obesity (Cairney, Hay, Faught, \& Hawes, 2005). Consequently, children with DCD experience difficulties with learning new motor skills and show delayed motor development (Jongmans, Smits-Engelsman, \& Schoemaker, 2003).

These connective consequences can be explained by the association; poor motor skills can lead to reduced motivation to participate in PA and reduced PA diminishes opportunities to improve a motor skill (McKenzie, Alcaraz, Sallis, \& Faucette, 1998). In other words, low participation in PA will impede motor skill development which, in turn, will lead to avoidance of PA participation (Larkin \& Rose, 2005).

Moreover, children with DCD often show high rates of social difficulties, low self-esteem, and associated behavioral problems during their childhood and adolescence (Ward \& Rodger, 2004). Therefore, poor motor skills of children with DCD can be linked to the low self-esteem and physical inactivity and these eventually causes diverse health problems (Straker et al., 2011).

\section{Motor skills learning in children with DCD}

The term „motor learning” refers to the relatively permanent behavioral changes associated with practice or experience (Schmidt \& Lee, 1988). According to Bo, Langan and Seidler (2008), motor learning can be divided into two different concepts: sensorimotor adaptation and sequence learning. The former indicates that people modify movements to environmental changes by using either sensory input or motor output characteristics. Whereas, sequence learning enables individuals to combine isolated movements into one smooth and coherent action. Significantly, due to poor sensorimotor adaptation and sequence learning, children with DCD often experience difficulties at smooth body movements or delay in motor learning and those comorbid problems are substantially overlapped with Attention Deficit Hyperactivity Disorder, Dyslexia, and Autism Spectrum Disorder (Kadesjo \& Gillberg, 1999).

Kirby and Sugden (2007) stated that "DCD is defined on the basis of a failure of the acquisition of skills in both gross and fine movements, which is not explicable on the basis of impaired general learning and similar exposure to the opportunity to gain motor skills as their peers". Children with DCD typically perform poor fine and/or gross motor skills, and consequently, their motor performances are usually slower, less accurate, and more variable than that of their peers (Zwicker, Missiuna, Harris, \& Boyd, 2012). Poor static and dynamic balance, postural control, manual catching and interceptive action, and sensory-motor coordination are representative motor skills deficits of children with DCD (Geuze, 2005; Piek \& Dyck, 2004; Wilson, Missiuna, Harris, \& Boyd, 2013).

Nonetheless, it has been proved that motor skills of children with DCD are likely to be improved by various interventions such as neuromotor task training, perceptual-motor and sensory-integration therapy, and kinesthetic training (Hillier, 2007; Schoemaker, Niemeijer, Reynders, \& Smits-Engelsman, 2003). These intervention approaches generally belong to either process-oriented or task-oriented approaches.

In spite of the efforts to develop effective intervention methods, the level of motor skills in children with DCD is still low compared to that of their peers. An updated review, investigating the effectiveness of newly approached movement-based interventions and the factors influencing the motor skills of children with DCD, is needed to provide the synthesized results of motor skills interventions for this population segment.

Therefore, the aim of the present study was to investigate the characteristics of effective movementbased interventions and to analyze influencing factors, including types of motor tasks, instructional strategies, and motor learning environment.

\section{Methods}

\section{Selection of studies}

The following electronic databases were used to search relevant studies published between January 1994 and December 2015: MEDLINE/PubMed, Science Direct, SPORTDiscus, PsycINFO, and ERIC (Education Resource Information Center). January 1994 and December 2015 were set as the date range for searching since in 1994 an international panel of experts was convened at a consensus meeting in London, 
Ontario, which accelerated the discussions and research on DCD.

Only studies written in English with full-text available were included. Key terms were divided into two different groups: diagnostic terms and the outcomes of interest indicating intervention type or method for motor learning. Subsequently, the following key terms were used: developmental coordination disorder, DCD, dyspraxia, coordination impairment, coordination problem/-s, coordination difficulty/-ies, coordination deficit/-s, movement skill problem/-s, movement deficit/-s, motor disorder, motor deficit/-s, motor difficulty/-ies, motor dysfunction, clumsiness, clumsy, motor function/-s disorder, $<$ AND $>$ exercise, physical activity, intervention, instruction, teaching, motor learning, motor development, acquisition, physical movement, skill improvement, training, practice, performance, movement outcome/-s, movement effect/-s.

Only studies with key terms in their title or abstract were included for review. To avoid omission of relevant studies, a motor learning expert repeatedly screened merged studies during the searching process.

\section{Inclusion and exclusion criteria}

Five inclusion criteria were set up based on PRISMA guidelines (Moher, Liberati, Tetzlaff, Altman, \& Prisma group, 2009):

1. The study should inform medical identification of DCD according to DSM-5 guidelines (APA, 2013) or previous version; DSM-IV (APA, 1994; APA, 2000). Additionally, it is considerable to include studies that used standardized tests to identify DCD.

2. Research design should be an experimental design such as randomized controlled trial (RCT) or quasi-experimental design with a control group and pre- and post-tests.

3. Participants should be 5 to 12 years of age at any level of DCD.

4. The intervention should be movement-based motor skills learning or sport-specific training with a defined period of or less than 12 weeks to minimize the non-treatment effect resulting from the natural growth of children.

5. The study should be published in a peerreviewed journal with full-text accessibility.

Meanwhile, four exclusion criteria were set up to avoid misinterpretation or over-representation of results:

1. Studies that include participants with identifiable neurological disorders (e.g., cerebral palsy) or other health conditions such as stroke, leukodystrophy, traumatic brain injury, autism spectrum disorder, language disorder, or muscular disorders were excluded.

2. Studies with mixed or multiple interventions design were excluded due to the likelihood of misinterpretation of the result.

3. Duplicated studies with interim data or same subject cohorts in multiple publications were excluded in order to eliminate over-representation of that particular result (Crowther, Lim, \& Crowther, 2010).

4. Studies with an unclear description of intervention were excluded (e.g., studies without specific tasks description, frequency, or duration).

Information on publication year and author, participant characteristics, sample size, gender ratio, identification method of DCD, study design, type and duration of intervention, motor skill assessment tool, and outcome measures were extracted.

\section{Results}

\section{Procedure of study selection}

A total of 317 studies were identified through preliminary e-database searching. After the screening process, only seven studies were selected as those fulfilled the inclusion criteria (Figure 1).

\section{Characteristics of included studies}

The characteristics of the included studies are described in Table 1. Among the articles analyzed, there was a total of 353 participants (children with DCD: 299, TD children: 54) with the age range of 5-12 years and the majority of participants was boys. Five studies used quasi-experimental study design, while only two studies used randomized controlled trial.

The most frequent method for assessment of the intervention effects was the Movement Assessment Battery for Children (MABC)-2/MABC and additional assessment tools were simultaneously used, including Trampoline Body Coordination Test, isokinetic machine and unilateral stance test, hand-held dynamometer, functional strength measure, Bruininks Oseretsky Test, and bilateral coordination and agility test.

The dominant setting in which the interventions were applied was the school. Also, interventions were applied to children under the supervision of motor learning experts in most of the included studies (Coetzee \& Venter, 2015; Ferguson, Jelsma, Jelsma, \& Smits-Engelsman, 2013; Giagazoglou, 


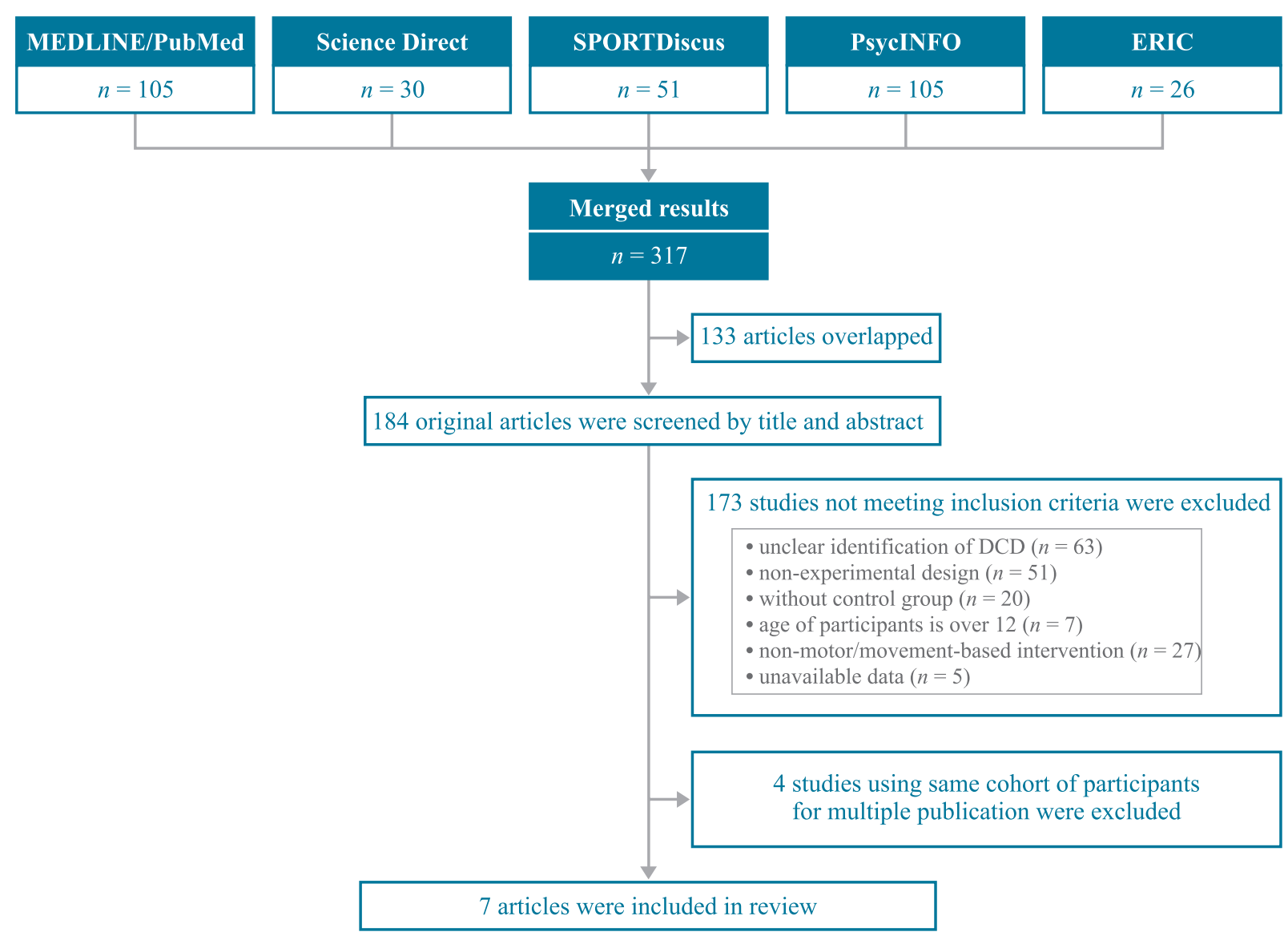

Figure 1. Flowchart of study selection

Sidiropoulou, Mitsiou, Arabatzi, \& Kellis, 2015; Jelsma, Geuze, Mombarg, \& Smits-Engelsman, 2014; Peens, Pienaar, \& Nienaber, 2008; Tsai, 2009). Whereas, Fong, Chung, Chow, Ma and Tsang (2013) used both institutional and home-based settings as children trained Taekwondo at the administering institute and home with a self-training curriculum..

\section{Effectiveness of motor skills interventions}

Ferguson et al. (2013) compared the effectiveness of Neuromotor Task Training (NTT) and Nintendo Wii Fit training. The Wii Fit intervention, witch consisted of practicing body movements according to the provided stimuli, showed improvements in fundamental motor skills even though the result were not statistically significant. The effect of Wii Fit training on motor skills improvement was repeatedly proved (Jelsma et al., 2014). Results showed that the balance of children with probable DCD was significantly improved after 6 week Wii Fit training, while no significant improvement was found in manual dexterity and aiming and catching.

The effect of martial arts training was also investigated. Fong et al. (2013) executed 12 weeks of Taekwondo training for children with DCD and the results proved that children in TKD group showed slower outcomes in a unilateral stance test that measured body sway velocity than children in the control group, but the equivocal effect was found in reactive balance control.

Meanwhile, Giagazoglou, Sidiropoulou, Mitsiou, Arabatzi and Kellis (2015) used the trampoline as a primary intervention tool. In spite of significant performance improvement in all balance post-tests after 12 weeks of balance training program, there were no statistically significant differences between the experimental and control group. However, the significant result was found in univariate group $\mathrm{x}$ time interaction effect of all 9 motor domains and in the total score of the Trampoline Body Coordination Test (TBCT), including segmental movement flow, body posture, 


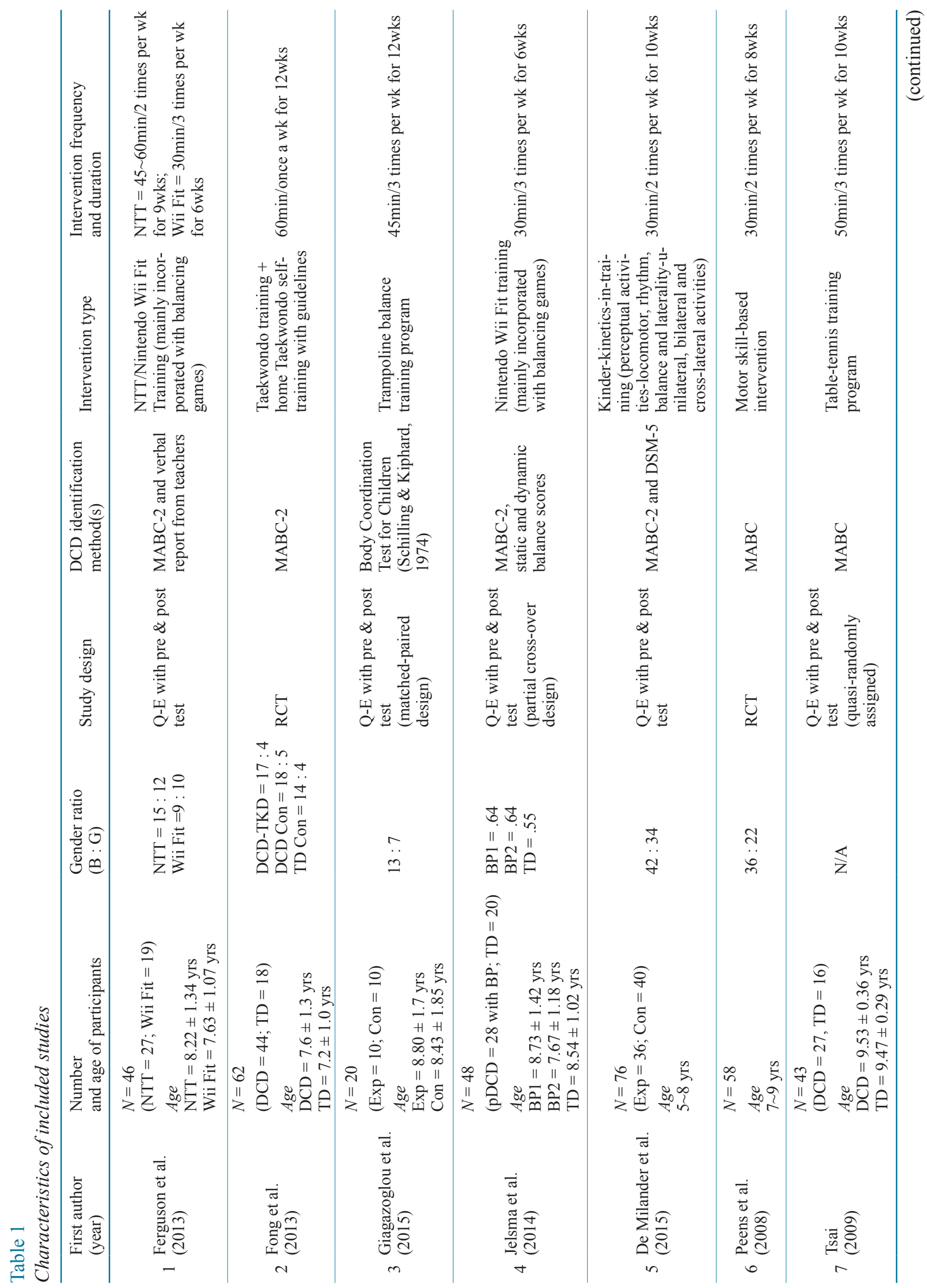




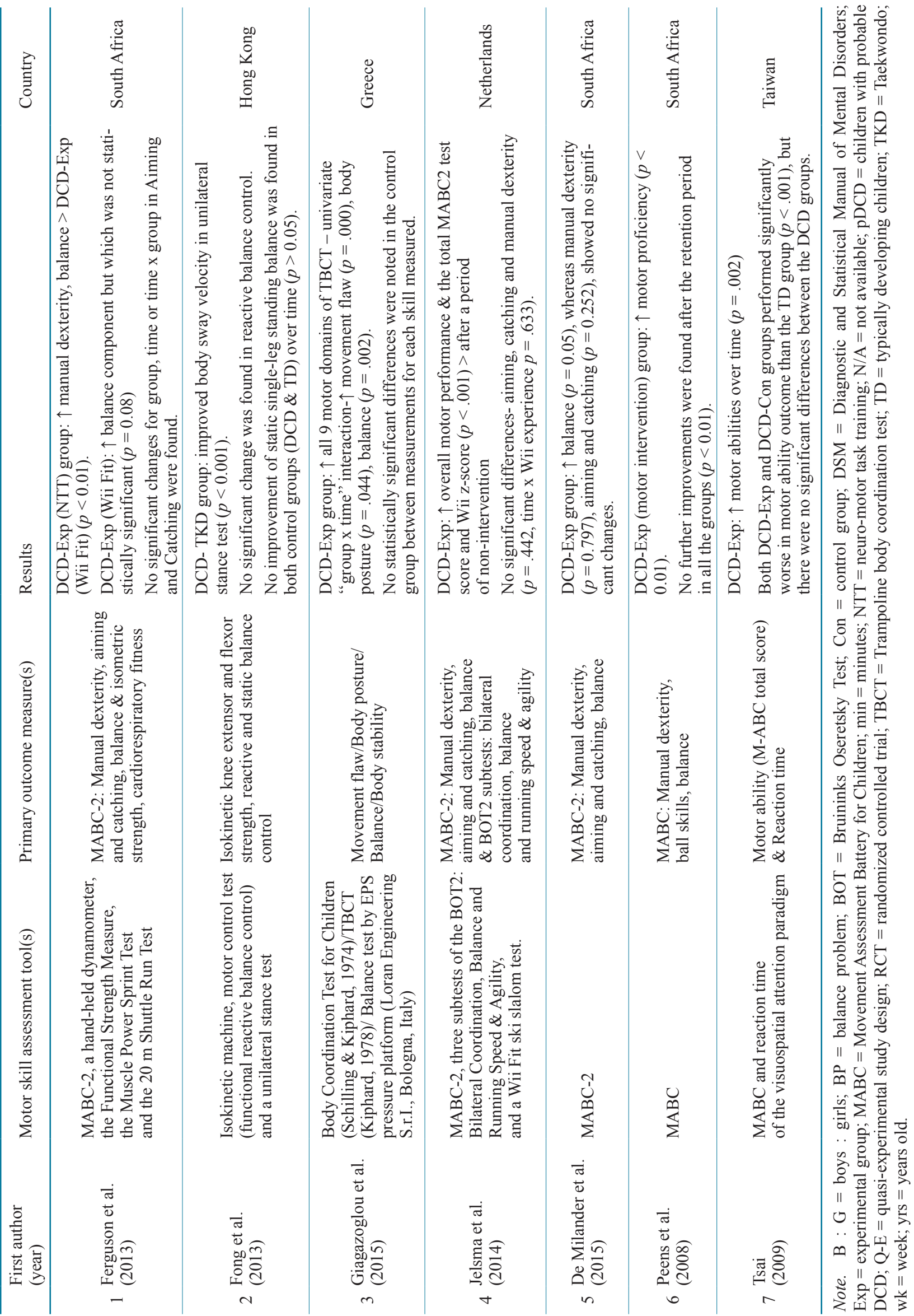


muscle strength, rhythm, balance, side asymmetry, body stability, and decreased redundant movements.

In a current study by de Milander, Coetzee and Venter (2015), the experimental group was trained using a perceptual-motor intervention program implemented by a Kinderkineticist who is familiar with the motor development of young children. Authors reported that the Kinderkinetics program as an early fundamental motor skills intervention positively influenced balance ability, whereas manual dexterity and aiming and catching showed no significant changes. In addition, there was no significant difference in the overall motor proficiency level between the experimental and control groups.

Similarly, Peens, Pienaar and Nienaber (2008) examined the motor skills-based intervention in addition to self-concept enhancing and other psychomotor interventions. After 8 weeks of the intervention period, children with DCD who endured a basic motor skills program showed greater improvement in fundamental motor skills than self-concept enhancing or psychomotor intervention groups. However, after a retention perion of two month, motor proficiency a showed no significant changes in all included groups.

Lastly, a ball-oriented intervention training was implemented by Tsai (2009). In this study, children with DCD were regularly trained in table tennis in a series of 50-min sessions for 10 weeks. The results demonstrated that the table tennis program significantly improved motor skills of the DCD-training group over time.

\section{Discussion}

From the review, motor/movement-based interventions using large muscles showed positive effects in improving motor skills of children with DCD even if the level of improvement was varied and some outcome measures resulted in equivocal effect. Present qualitative review study investigated characteristics of the various types of fundamental and sport-specific motor skills interventions and examined the influential factors enhancing motor skills.

It has been generally accepted that due to the motor deficiencies such as poor predictive control, rhythmic coordination and timing within and between limbs, and executive function, children with DCD often struggle with participating in PA and learning new motor skills (Wilson, Ruddock, Smits-Engelsman, Polatajko, $\&$ Blank, 2013). As such, the low level of PA and poor motor abilities have been manifested among children with DCD (Batey et al., 2014; Cairney, Hay, Faught, Wade, et al., 2005; Straker et al., 2011).

Nevertheless, the findings of the present review suggest that motor skills interventions focusing on gross motor movement can be recommended to improve motor performance of children with DCD. Even though the required time for motor skills acquisition to children with DCD is unsurprisingly higher than TD children (Missiuna, 1994), it should be highlighted that developmentally appropriate motor skills interventions have high potential to reduce disparities in motor skills development among this population segment. In other words, it is critical to consider various motor skill capacities and developmental maturity of children with DCD when designing instructional environment (Graham, Castenada, Hopple, Manross, \& Sanders, 1992).

In review, the type of interventions was categorized as task-oriented interventions, including fundamental motor skills and video game-based Wii Fit training, or sport-specific interventions, including Taekwondo and a Table tennis program. The task-oriented interventions are generally aimed at practicing specific motor skills that child struggles with. This type of intervention has shown the largest effect size compared to other intervention approaches (e.g., process-oriented and physical therapy and chemical supplements) (Harris, Mickelson, \& Zwicker, 2015) and similar results were shown in review.

Interestingly, the video game-based interventions in pursuit of involving preferable activities by children with DCD were newly examined. In fact, the impact of the Wii Fit training program on balance has been proved in adult populations by a few studies (Bieryla \& Dold, 2013; Fung, Ho, Shaffer, Chung, \& Gomez, 2012; Nitz, Kuys, Isles, \& Fu, 2010). Its beneficial training effect was also found in balance and overall motor performance among children with DCD (Ferguson et al., 2013; Jelsma et al., 2014). However, one of the findings showed an equivocal result due to the lack of statistical significance (Ferguson et al., 2013). Therefore, more studies on a large scale are needed to support the effect of Wii Fit training on balance in children with DCD. The possible reasoning of the Wii Fit program's balance-enhancing effect is because it leads players to utilize visuomotor coordination and balance by reacting to constantly changing stimuli while watching the screen (Ferguson et al., 2013).

Taekwondo training, once a week, showed no positive changes in reactive balance control in children with DCD (Fong et al., 2013) while other studies 
reported the improved results in balance by participating in intervention programs 2 to 3 times per week, depending on the study design. This finding is in line with the relationship between frequency and training effect; higher frequency, better effect given the intensity is controlled (Delisle, Werch, Wong, Bian, \& Weiler, 2010; Nieuwland et al., 2000). Nonetheless, we should interpret this finding with caution as the effect of motor skills intervention can be influenced by intensity and duration of intervention and other external factors (e.g., instructor/therapist proficiency of teaching skills, quality of the program, and parental intervention). Still, an intervention strategy with daily tasks for children with DCD is recommended (Harris et al., 2015).

We found a noticeable tendency in the included studies in which the school was uniformly set for providing intervention even though the tasks given to children with DCD were varied. This similarity in the learning environment can be interpreted as the convenience of intervention execution since monitoring each child at his/her home is time-consuming and can reduce reliability of findings due to the heterogeneity of each individual's home environment.

Lastly, only one study (Peens et al., 2008) measured retention effect after 2 months of intervention completion, which enables researchers to reflect the sustainability of changes. No further improvements were found after the retention period in the motor skills intervention group (Peens et al., 2008). More studies with retention tests should be further analyzed to verify the sustainability of the effect of motor skills interventions.

\section{Limitations}

In spite of countless published studies focused on children with DCD, the studies based on motor skills intervention with pre- and post-test design are still lacking. Consequently, the present review study faced limitations such as low number of included studies and small total sample size of target population, which may yield the interpretive bias.

Included studies provided data in motor skills outcomes and specific type of intervention with duration and frequency. However, it is still unclear how intensively the interventions were provided and how external variables (e.g., parental intervention or quality of instruction) were controlled.

In addition, due to the excessive level of methodological and clinical heterogeneity of interventions, it was impossible to draw synthesized data of outcome measures. Furthermore, since there was no effort made to examine the relationship between improved motor skills and other health-related outcomes among children with DCD, it is questionable to state that the motor skills interventions genuinely assists those children with DCD in improving other physical, behavioural, and functional outcomes.

Thus, more rigorous intervention studies with experimental study design, investigating the relationship between motor skills and daily activities, the sustainability of changes, and the impact of the intervention on other physical, health, social and emotional outcomes should be conducted. Also, it is necessary to specifity the clear methodology and intervention description to provide more valid evidence-based motor skills intervention for children with DCD.

\section{Perspective}

Various types of motor/movement-based interventions can improve motor skills in children with DCD. In spite of the differences in duration, frequency, and period among interventions, the majority of studies demonstrated the promising effect in improving motor performance of children with DCD.

Nonetheless, studies with pre- and post-test experimental design in which the researchers can assess more accurate and clearer changes in outcome measures, are still lacking. In addition, there are limited studies reporting the retention effect, intensity of intervention, and relationship between motor skills and other daily activities. Consequently, it is difficult to provide clear guidelines for children with DCD in order to sustainably improve various motor skills and other health outcomes.

Therefore, more rigorous intervention studies are needed to:

1. identify motor intervention best practices,

2. examine the sustainability of changes, and

3. examine the impact of the intervention on other physical, health, social, and emotional outcomes.

\section{Acknowledgement}

The study was made in the frame of the project IGA FTK_2015_001 financially supported by the Palacký University Science Foundation.

\section{References}

American Psychiatric Association. (1994). DSM-IV: Diagnostic and statistic manual of mental disorders. Washington, DC: American Psychiatric Association. 
American Psychiatric Association. (2013). Diagnostic and statistical manual of mental disorders (5th ed.). Arlington, VA: American Psychiatric Publishing.

American Psychiatric Association. (2000). Diagnostic and Statistical Manual of Mental Disorders. 4th text revision ed. Washington, DC: American Psychiatric Association, 553-557.

Barnhart, R. C., Davenport, M. J., Epps, S. B., \& Nordquist, V. M. (2003). Developmental coordination disorder. Physical Therapy, 83(8), 722.

Batey, C. A., Missiuna, C. A., Timmons, B. W., Hay, J. A., Faught, B. E., \& Cairney, J. (2014). Self-efficacy toward physical activity and the physical activity behavior of children with and without Developmental Coordination Disorder. Human Movement Science, 36, 258-271.

Bieryla, K. A., \& Dold, N. M. (2013). Feasibility of Wii Fit training to improve clinical measures of balance in older adults. Clin Interv Aging, 8(1), 775-781.

Blank, R., Smits-Engelsman, B., Polatajko, H., \& Wilson, P. (2012). European Academy for Childhood Disability (EACD): Recommendations on the definition, diagnosis and intervention of developmental coordination disorder (long version). Developmental Medicine \& Child Neurology, 54(1), 54-93.

Bo, J., Langan, J., \& Seidler, R. (2008). Cognitive Neuroscience of Skill Acquisition. Advances In Psychology, 139 (Human Learning), 101-112. doi:10.1016/S0166-4115(08)10009-7.

Cairney, J., Hay, J. A., Faught, B. E., \& Hawes, R. (2005). Developmental coordination disorder and overweight and obesity in children aged 9-14 y. International Journal of Obesity, 29(4), 369-372.

Cairney, J., Hay, J. A., Faught, B. E., Wade, T. J., Corna, L., \& Flouris, A. (2005). Developmental coordination disorder, generalized self-efficacy toward physical activity, and participation in organized and free play activities. The Journal of Pediatrics, 147(4), 515-520.

Coetzee, P., Venter, E. H. (2015). Recent advances in knowledge of BTV-host-vector interaction. Veterinaria italiana, 51(4), 301-311.

Crowther, M., Lim, W., Crowther, M. A. (2010). Systematic review and meta-analysis methodology. Bood, 116(17), 3140-3146.

De Milander, M., Coetzee, F. F., \& Venter, A. (2015). Perceptual-motor intervention for developmental coordination disorder in grade 1 children. South African Journal for Research in Sport, Physical Education \& Recreation (SAJR SPER), 37(2).

Delisle, T. T., Werch, C. E., Wong, A. H., Bian, H., \& Weiler, R. (2010). Relationship between frequency and intensity of physical activity and health behaviors of adolescents. Journal of School Health, 80(3), 134-140.

Deng, S., Li, W. G., Ding, J., Wu, J., Zhang, Y., Li, F., \& Shen, X. (2013). Understanding the mechanisms of cognitive impairments in developmental coordination disorder. Pediatric Research, 75(1-2), 210-216.

Dewey, D., Kaplan, B. J., Crawford, S. G., \& Wilson, B. N. (2002). Developmental coordination disorder: associated problems in attention, learning, and psychosocial adjustment. Human Movement Science, 21(5), 905-918.

Faught, B. E., Hay, J. A., Cairney, J., \& Flouris, A. (2005). Increased risk for coronary vascular disease in children with developmental coordination disorder. Journal of Adolescent Health, 37(5), 376-380.

Ferguson, G. D., Jelsma, D., Jelsma, J., \& Smits-Engelsman, B. C. M. (2013). The efficacy of two task-orientated interventions for children with Developmental Coordination Disorder: Neuromotor Task Training and Nintendo Wii Fit training. Research in Developmental Disabilities, 34(9), 2449-2461.

Fong, S. S., Chung, J. W., Chow, L. P., Ma, A. W., \& Tsang, W. W. (2013). Differential effect of Taekwondo training on knee muscle strength and reactive and static balance control in children with developmental coordination disorder: A randomized controlled trial. Research in Developmental Disabilities, 34(5), 1446-1455.

Fung, V., Ho, A., Shaffer, J., Chung, E., \& Gomez, M. (2012). Use of Nintendo Wii Fit ${ }^{\mathrm{TM}}$ in the rehabilitation of outpatients following total knee replacement: a preliminary randomised controlled trial. Physiotherapy, 98(3), 183-188.

Geuze, R. H. (2005). Postural control in children with developmental coordination disorder. Neural Plast, 12, 183-196.

Giagazoglou, P., Sidiropoulou, M., Mitsiou, M., Arabatzi, F., \& Kellis, E. (2015). Can balance trampoline training promote motor coordination and balance performance in children with developmental coordination disorder? Research in Developmental Disabilities, 36, 13-19.

Gilger, J. W., \& Kaplan, B. J. (2001). Atypical brain development: a conceptual framework for understanding developmental learning disabilities. Developmental Neuropsycho$\log y, 20(2), 465-481$.

Graham, G., Castenada, R., Hopple, C., Manross, M., \& Sanders, S. (1992). Developmentally appropriate physical education practices for children. Reston, VA: National Association for Sport and Physical Education, Council on Physical Education for Children.

Harris, S. R., Mickelson, E. C., \& Zwicker, J. G. (2015). Diagnosis and management of developmental coordination disorder. Canadian Medical Association journal = journal de l'Association medicale canadienne, 187(9), 659-665.

Hillier, S. (2007). Intervention for children with developmental coordination disorder: a systematic review. Internet Journal of Allied Health Sciences and Practice, 5(3), 7.

Jelsma, D., Geuze, R. H., Mombarg, R., \& Smits-Engelsman, B. C. (2014). The impact of Wii Fit intervention on dynamic balance control in children with probable Developmental Coordination Disorder and balance problems. Human Movement Science, 33, 404-418.

Jongmans, M. J., Smits-Engelsman, B. C., \& Schoemaker, M. M. (2003). Consequences of comorbidity of developmental coordination disorders and learning disabilities for severity and pattern of perceptual-motor dysfunction. Journal of Learning Disabilities, 36(6), 528-537.

Kadesjo, B., \& Gillberg, C. (1999). Developmental coordination disorder in Swedish 7-year-old children. Journal of the American Academy of Child \& Adolescent Psychiatry, 38(7), 820-828.

Kiphard, E. J. (1978). Testanalysen zum trampolinkörperkoordinationstest (TKT). Psychomotorik, 2(3), 111-114.

Kirby, A., \& Sugden, D. A. (2007). Children with developmental coordination disorders. Journal of the Royal Society of Medicine, 100(4), 182-186.

Kwan, M. Y., Cairney, J., Hay, J. A., \& Faught, B. E. (2013). Understanding physical activity and motivations for children 
with Developmental Coordination Disorder: An investigation using the Theory of Planned Behaviour. Research in developmental disabilities, 34(11), 3691-3698.

Larkin, D., \& Rose, E. (2005). Assessment of developmental coordination disorder. In D. Sugden \& M. Chambers (Eds.), Children with developmental coordination disorder (pp. 135-154). London: Whurr.

Lichtenstein, P., Carlström, E., Råstam, M., Gillberg, C., \& Anckarsäter, H. (2010). The genetics of autism spectrum disorders and related neuropsychiatric disorders in childhood. American Journal of Psychiatry, 167(11), 1357-1363.

McKenzie, T. L., Alcaraz, J. E., Sallis, J. F., \& Faucette, E. N. (1998). Effects of a physical education program on children's manipulative skills. Journal of Teaching in Physical Education, 17, 327-341.

Missiuna, C. (1994). Motor skill acquisition in children with developmental coordination disorder. Adapted Physical Activity Quarterly, 11(2), 214-235.

Moher, D., Liberati, A, Tetzlaff, J., Altman, D. G., \& Prisma Group (2009). Preprint-preferred reporting intems for systematic reviews and meta analyses: the PRISMA statement. PLoS med, 6(7), e1000097.

Nieuwland, W., Berkhuysen, M. A., van Veldhuisen, D. J., Brügemann, J., Landsman, M. L., van Sonderen, E., ... \& Rispens, P. (2000). Differential effects of high-frequency versus low-frequency exercise training in rehabilitation of patients with coronary artery disease. Journal of the American College of Cardiology, 36(1), 202-207.

Nitz, J. C., Kuys, S., Isles, R., \& Fu, S. (2010). Is the Wii Fit ${ }^{\mathrm{TM}}$ a new-generation tool for improving balance, health and well-being? A pilot study. Climacteric, 13(5), 487-491.

Peens, A., Pienaar, A. E., \& Nienaber, A. W. (2008). The effect of different intervention programmes on the self-concept and motor proficiency of 7-to 9-year-old children with DCD. Child: Care, Health and Development, 34(3), 316-328.

Piek, J. P., \& Dyck, M. J. (2004). Sensory-motor deficits in children with developmental coordination disorder, attention deficit hyperactivity disorder and autistic disorder. Human Movement Science, 23(3), 475-488.

Rasmussen, P., \& Gillberg, C. (2000). Natural outcome of ADHD with developmental coordination disorder at age 22 years: a controlled, longitudinal, community-based study. Journal of the American Academy of Child \& Adolescent Psychiatry, 39(11), 1424-1431.

Schilling, F., \& Kiphard, E. J. (1974). The whole body coordination test for children. Manual.

Schmidt, R. A., \& Lee, T. (1988). Motor control and learning. Champaign, IL: Human kinetics.

Schoemaker, M. M., Niemeijer, A. S., Reynders, K., \& SmitsEngelsman, B. C. (2003). Effectiveness of neuromotor task training for children with developmental coordination disorder: a pilot study. Neural Plasticity, 10(1-2), 155-163.

Straker, L. M., Campbell, A. C., Jensen, L. M., Metcalf, D. R., Smith, A. J., Abbott, R. A., ... Piek, J. P. (2011). Rationale, design and methods for a randomized and controlled trial of the impact of virtual reality games on motor competence, physical activity, and mental health in children with developmental coordination disorder. BMC Public Health, 11, 654.

Tsai, C. L. (2009). The effectiveness of exercise intervention on inhibitory control in children with developmental coordination disorder: Using a visuospatial attention paradigm as a model. Research in Developmental Disabilities, 30(6), $1268-1280$

Ward, A., \& Rodger, S. (2004). The application of cognitive orientation to daily occupational performance (CO-OP) with children 5-7 years with developmental coordination disorder. The British Journal of Occupational Therapy, 67(6), 256-264.

Wilson, P. H., \& McKenzie, B. E. (1998). Information processing deficits associated with developmental coordination disorder: A meta-analysis of research findings. Journal of Child Psychology and Psychiatry, 39(6), 829-840.

Wilson, P. H., Ruddock, S., Smits-Engelsman, B., Polatajko, H., \& Blank, R. (2013). Understanding performance deficits in developmental coordination disorder: a meta-analysis of recent research. Developmental Medicine \& Child Neurology, 55(3), 217-228.

Zwicker, J. G., Missiuna, C., Harris, S. R., \& Boyd, L. A. (2012). Developmental coordination disorder: a review and update. European Journal of Paediatric Neurology, 16(6), 573-581.

\section{Corresponding author \\ Daehyoung Lee \\ Email address | lee2055@indiana.edu}

